
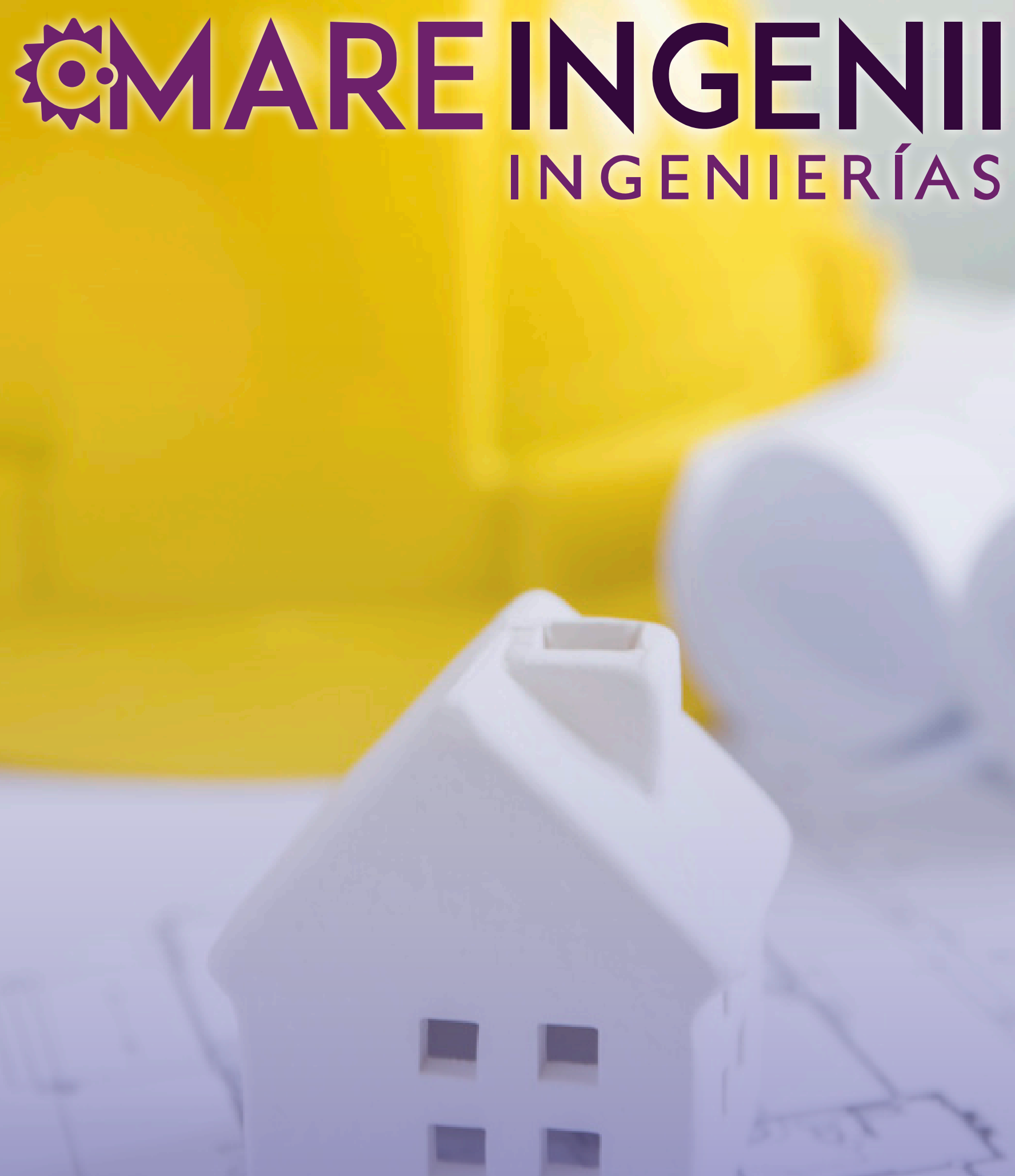

Ingeniería en Salud y Seguridad para el Trabajo (SST) 


\title{
Simulación de colchoneta que alterna puntos de apoyo para pacientes con movilidad reducida
}

\section{Mattress simulation that alternate support points for patients with reduced mobility}

\author{
Diana Paola Gómez Ariza'; Luisa Fernanda Melo Cajamarca²
}

'Especialista en bioingeniería de la Universidad Distrital Francisco José de Caldas, licenciada en física y docente de áreas básicas de la Fundación Universitaria San Mateo

${ }^{2}$ Profesional en Ingeniería Electrónica de la Universidad Distrital Francisco José de Caldas. Especialista en Bioingeniería de la Universidad Distrital Francisco José de Caldas.

Como citar: Gómez Ariza, D; Melo Cajamarca, L. 2019. Simulación de colchoneta que alterna puntos de apoyo para pacientes con movilidad reducida. Mare Ingenii. Ingenierías 1(1). Disponible en http://cipres.sanmateo.edu.co/index.php/mi

Recibido: Agosto 10 de 2019

Aceptado: Septiembre 11 de 2019

\section{RESUMEN}

La movilidad reducida representa un importante problema de salud, facilita el desarrollo de enfermedades en el paciente y eleva los costos del sistema sanitario. Esto, debido a que aumenta el tiempo de hospitalización, la demanda de horas de enfermería para estar girando al paciente y curando las heridas, la prescripción de antisépticos y antibióticos, entre otros. Desde 2003 hasta 2010 el Ministerio de la Protección Social en Colombia gastó 22.797.600.000 pesos colombianos en pacientes con discapacidad, de los cuales una cantidad significativa fue destinado a las personas con movilidad reducida. Una ayuda factible para estos pacientes es la disminución de presión en sus puntos de apoyo cuando están acostados o sentados, por medio de una colchoneta que alterne dichos puntos mediante un sistema de inflado-desinflado de cilindros, redistribuyendo periódicamente la presión, imitando el efecto de los movimientos espontáneos para conservar la perfusión tisular. Este artículo expone una simulación del sistema colchón-paciente a través de un software que reduce diseño, tiempo y costos, disminuye la cantidad de prototipos de prueba antes de iniciar con una línea de producción y ayuda con estudios de calidad y seguridad de nuevos diseños, generando resultados con alto nivel de confiabilidad.

Palabras clave: Simulación; colchoneta; Movilidad reducida. 


\section{ABSTRACT}

Reduced mobility is a major health problem because it facilitates the development of disease in the patient and increases the cost of the health system. It increases the length of hospitalization, the demand for nursing hours for turning the patient and wound healing, the prescription of antiseptics and antibiotics, and so on. From 2003 to 2010, the Ministry of Social Protection in Colombia spent 22,797,600,000 colombian pesos in disabled patients, of which a significant amount went to people with reduced mobility. A possible help for these patients is the change of support points when lying down or sitting, given by a mattress that alternates these points through a system of inflation-deflation of cylinders, redistributing pressure periodically, mimicking the effect of spontaneous movement to preserve tissue perfusion. This paper exposes a mattresspatient system simulation through software, which reduces design, time and costs, decreases the amount of testing prototypes before starting a production line, and help to do studies on quality and safety of new designs producing results with high reliability.

Keywords: Simulation, mattress, reduced mobility.

\section{INTRODUCCIÓN}

El movimiento es fundamental en la vida de los seres humanos, cuando se pierde trae como consecuencia manifestaciones tales como disminución en la circulación sanguínea. Así, aumenta la probabilidad de coagulación dentro de los vasos sanguíneos, ocasionando trombosis venosa profunda y/o tromboembolismo pulmonar. Por otra parte, la pérdida de movimiento puede ocasionar colapso de alveolos pulmonares desarrollando atelectasia.La discapacidad afecta a mil millones de personas en el mundo, de las cuales tres de cada cuatro presentan movilidad reducida [1]. En Colombia, la movilidad reducida afecta al 0,83\% de la población [2]. La movilidad reducida trae consigo úlceras por presión, que alcanzan una prevalencia en población hospitalizada desde 3,5\% hasta el 29\%, variando según las series estudiadas. Por ejemplo, en el Hospital Universitario San Ignacio de Bogotá, se observó el 18\% de esta prevalencia [3], [4].Cuando el paciente se encuentra sobre una colchoneta experimenta una fuerza de contacto como consecuencia de su peso, generando una presión. Si dicha presión es mayor de 32 mm Hg sobre el tejido de la piel, ocluirá el flujo sanguíneo capilar en los tejidos blandos, provocando una hipoxia y si no se cambia la posición, puede llegar a una necrosis de los mismos. Se sabe que el 95\% de las úlceras por presión que presentan los pacientes son evitables [4].

Para atender a los pacientes con movilidad reducida existen varias estrategias. Cuando la persona se encuentra hospitalizada, el grupo de enfermería se encarga de cambiarlo de posición cada dos o cuatro horas [3], elevando la demanda de horas de enfermería para estar girando al paciente y curando las heridas. Además, las complicaciones de la movilidad reducida aumentan el tiempo de hospitalización pues es necesario tratarlas. Se incrementa también la prescripción de antisépticos y antibióticos, entre otros. Estas estrategias elevan los costos del sistema sanitario que durante el periodo de 2003 a 2010, según el Ministerio de la Protección Social de Colombia [5], gastó 22.797.600.000 de pesos colombianos en pacientes con discapacidad, de los cuales una cantidad significativa se asignó para la atención de pacientes con movilidad reducida. Existe otro mecanismo de ayuda que es ubicar al paciente en un colchón antiescaras que busca disminuir la presión continua sobre las prominencias óseas, permitiendo una correcta circulación sanguínea. Este tipo de colchón está indicado para todos aquellos pacientes inmovilizados que puedan presentar sudoración excesiva, incontinencia vesical o fecal, alteraciones en el flujo sanguíneo, fracturas o problemas neurológicos.

Otra alternativa que se ha planteado es a través del inflado y desinflado alternante de las celdas (también llamada terapia activa) de forma que alivia eficazmente la presión por debajo de límites. La terapia ha sido diseñada para imitar los efectos de los movimientos espontáneos del cuerpo, permitiendo la perfusión tisular al máximo. Los estudios clínicos demuestran, continuamente, resultados excelentes para los pacientes más vulnerables o los que tienen heridas. El compresor silencioso y sin vibraciones interactúa con el sensor Auto-Matt, ubicado en el colchón, para realizar un ajuste automático de la presión en función del volumen y ubicación de cada paciente [6].

El presente trabajo realiza la simulación de un sistema colchoneta-paciente, alternando cambios en los puntos de apoyo donde se distribuye la fuerza ejercida por el cuerpo sobre la superficie de contacto. La simulación reduce el tiempo y costos 
de diseño; adicionalmente disminuye la cantidad de prototipos físicos de prueba antes de iniciar con una línea de producción y ayuda con los estudios de calidad y seguridad de nuevos diseños, generando resultados con un alto nivel de confiabilidad.

\section{- Posiciones anatómicas y puntos de riesgo}

Las posiciones anatómicas con mayores puntos de riesgo generados por movilidad reducida son: posición decúbito (supino, lateral y prono) al igual que sentado.
Decúbito supino. En la posición de decúbito supino, la localización más frecuente de las úlceras por presión son: talones, sacro, codos, omóplatos y zona occipital.

Decúbito lateral. En la posición de decúbito lateral, la localización de las úlceras son: orejas, acromion, codos, caderas, cóndilos y maléolos.

Figura 1. Puntos de riesgo en la posición decúbito supino.Fuente: Protocolo de actuación en ulceras por presión [7].

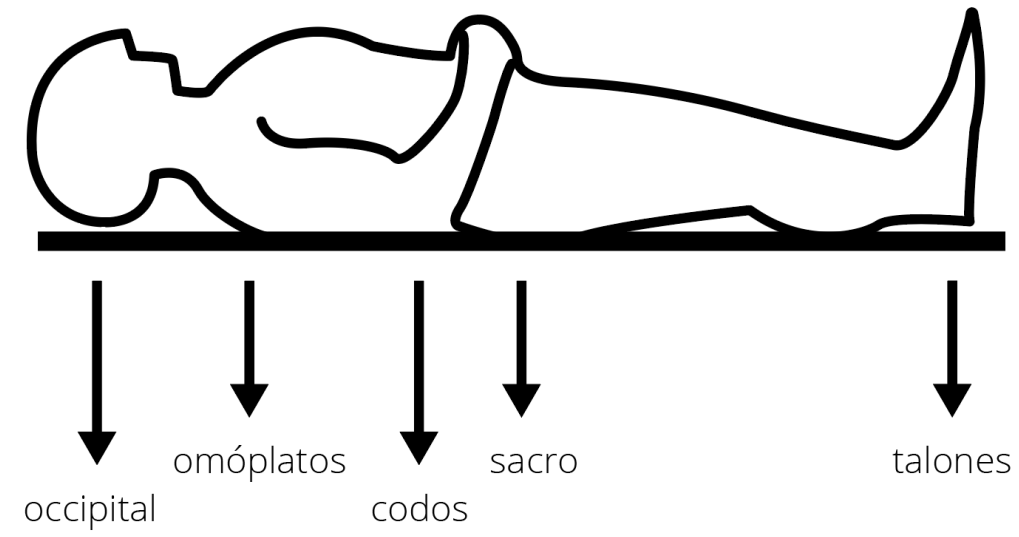

Figura 2. Puntos de riesgo en la posición decúbito lateral. Fuente: Protocolo de actuación en ulceras por presión [7]

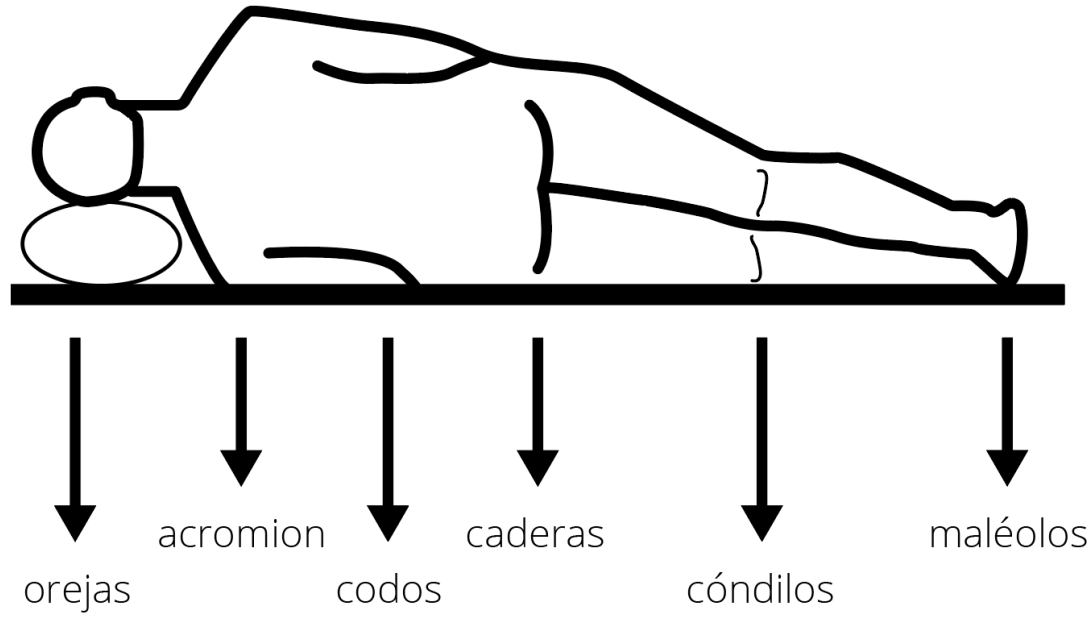


Decúbito prono. En la posición de decúbito prono: dedos de los pies, rodillas, espinas ilíacas, órganos genitales en hombres, mamas en mujeres, costillas, mejilla y oreja.
Sentado. En otras posiciones, como la de sentado, los puntos más susceptibles para la aparición de úlceras son los omóplatos, codos, coxis, subglúteos, huecos, poplíteos, gemelos y talones.

Figura 3. Puntos de riesgo en la posición decúbito prono. Fuente: Protocolo de actuación en ulceras por presión, [7].

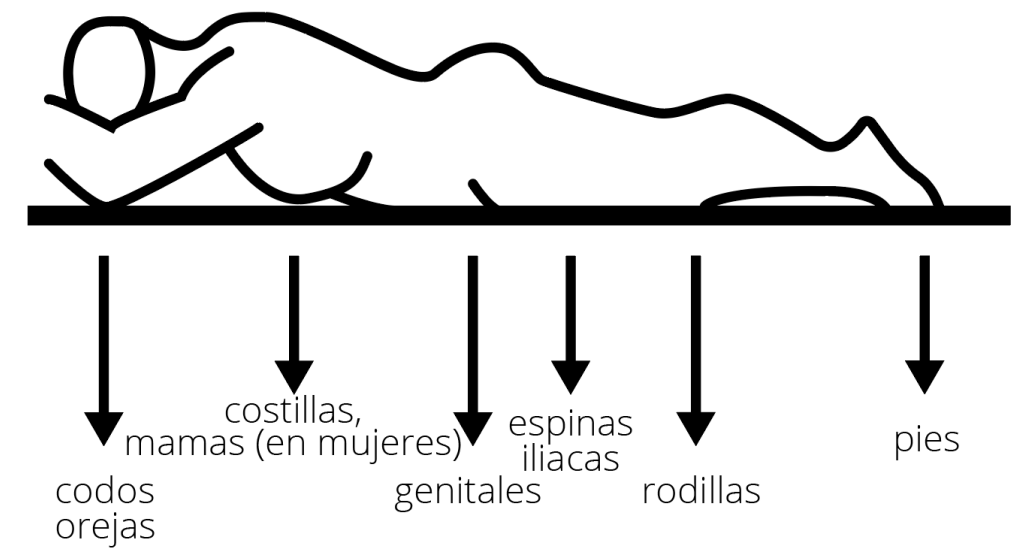

Figura 4. Puntos de riesgo en la posición sentado. Fuente: Protocolo de actuación en ulceras por presión, [7].

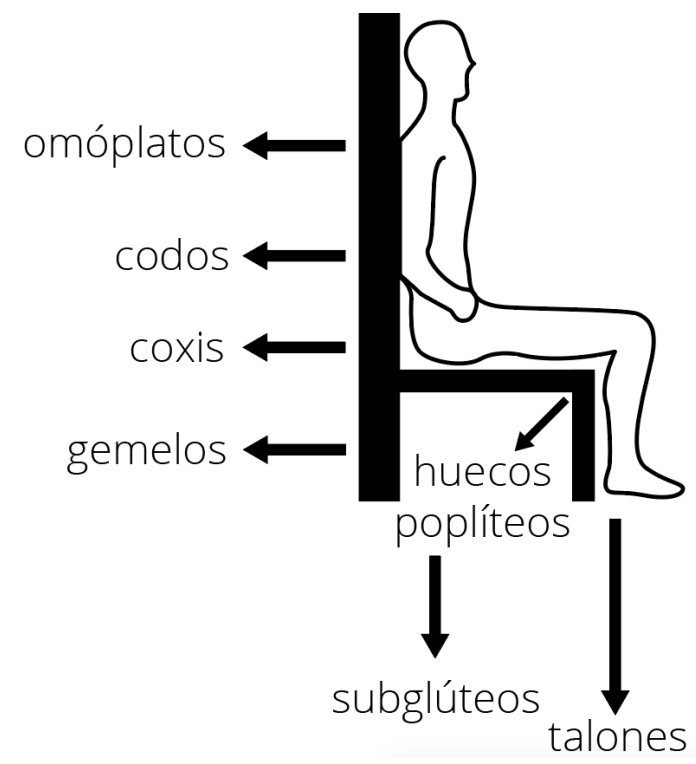




\section{METODOLOGÍA}

\section{- Software}

Existen software especializados en modelamiento, animación y simulación. Entre los más significativos están: Rhino (McNeel, Seattle, USA), Autocad (Autodesk Inc., California, USA), Blender (herramienta gratuita), Solid Edge (Autodesk Inc., California, USA), Solid Works (Dessault Systemes, Massachusetts,USA) y Catia (Dassault Systemes, Massachusetts, USA).

Se encontraron varios inconvenientes para trabajar con algunos programas anteriormente mencionados por las restricciones que presentaban. Dentro de las más relevantes se encuentran: no permitir simular el sistema colchoneta-paciente, lista de materiales reducida sin opción de modificar parámetros. El software Catia y SolidWorks realizan modelamiento de estructuras bajo diseños rigurosos en términos de medidas y materiales. También permiten simular diferentes estados de fuerzas puntuales y distribuidas en diseños de una sola pieza o ensambladas. Adicionalmente, presentan como ventaja la posibilidad de simulación y facilidad de manejo de los módulos. Estas características permiten los parámetros de materiales y propiedades físicas. SolidWorks permite elaborar la simulación dejando el cuerpo sobre la colchoneta, lo que obliga a trabajar puntos señalados con fuerzas distribuidas. Sin embargo, no es software libre, es decir, requiere licencia; la manipulación de la plataforma no es básica por lo que se recomienda tener conocimientos previos en programas similares de diseño.
El software seleccionado para el desarrollo del trabajo es SolidWorks (2010), de la empresa Dassault Systemes SolidWorks Corp., ubicada en Massachusetts, Estados Unidos. Las características presentadas por este software se acoplaron a las necesidades presentadas para la elaboración de la simulación, razón principal para su uso, además de contar con licencia empresarial para su aplicación y conocimientos previos sobre su manejo.

Solidworks contiene dos módulos de simulación incorporados Ilamados FlowSimulation y Solidworks simulation. El primero es utilizado para realizar simulación con fluidos y aire. El segundo modulo permite realizar simulación de fuerzas, deformación de materiales expuestos a fuerzas externas, entre otras.

\section{- Parámetros y medidas de la colchoneta}

Para poder realizar la simulación de la colchoneta se plantean como prioridad los parámetros de elaboración como lo son las dimensiones, puntos de contacto, material a utilizar y sistema de alimentación neumático. Actualmente el material de recubrimiento más utilizado en la fabricación de colchones y colchonetas para uso clínico es Plasti Tex Gris Aluminizado. Este es una película de $80 \%$ de policloruro de vinilo (PVC), que contiene $20 \%$ de algodón poliéster. Además, presenta densidad de $150 \mathrm{~kg} / \mathrm{m}^{3}$. Este material permite una fácil limpieza al ser impermeable y mantener libre de ácaros [8].

El material seleccionado del software es Delrin 2700 NC010, copolimero de acetal de baja viscosidad, plástico que presenta especificaciones similares al utilizado como recubrimiento de colchones de la línea médica [9]. Las especificaciones, mencionadas con anterioridad, se ajustaron dentro de la simulación y son mencionadas en la Tabla 1.

Tabla 1. Parámetros del material a utilizar en la simulación.Fuente: Elaboración propia

\begin{tabular}{|c|c|c|}
\hline Nombre de propiedad & Valor & Unidades \\
\hline Módulo elástico & $2.9 \mathrm{e}+009$ & $\mathrm{~N} / \mathrm{m}^{\wedge} 2$ \\
\hline Coeficiente de Poisson & 0.3 & NA \\
\hline Densidad & 141 & $\mathrm{~kg} / \mathrm{m}^{\wedge} 3$ \\
\hline Límite de tracción & $4.07 \mathrm{e}+007$ & $\mathrm{~N} / \mathrm{m}^{\wedge} 2$ \\
\hline Límite elastic & $6.3 e+007$ & $\mathrm{~N} / \mathrm{m}^{\wedge} 2$ \\
\hline $\begin{array}{l}\text { Factor de endurecimiento }(0.0-1.0 ; 0.0=\text { isotrópico; } \\
1.0=\text { cinemático })\end{array}$ & 0.85 & NA \\
\hline
\end{tabular}


Luego de ajustar las especificaciones del material, se consideraron las medidas de las camas hospitalarias. Sus dimensiones se ajustaron a la colchoneta de la siguiente manera: $2.25 \mathrm{~m}$ de largo, $90 \mathrm{~cm}$ de ancho y $9 \mathrm{~cm}$ de grosor. Adicionalmente, es importante no sobrepasar los $17 \mathrm{~cm}$ de espesor, después de este grosor ya no se considera como colchoneta [10].
- Variabilidad de puntos de apoyo

Primero, se implementa el sistema neumático de alimentación, adicionando una base de espuma rectangular plana de $4 \mathrm{~cm}$ de espesor, atravesada por ductos que transportan el aire. Segundo, se busca alternar los puntos de apoyo con la superficie. Por ello, en la parte superior de la base de espuma, se adapta un sistema de cilindros vacíos de $14 \mathrm{~cm}$ de diámetro y $5 \mathrm{~cm}$ de espesor que permiten la entrada y salida de aire por medio de los ductos.

Figura 5. Muestra el sistema de ductos que atraviesan la espuma para el bombeo de aire. Fuente:

\section{Elaboración propia}

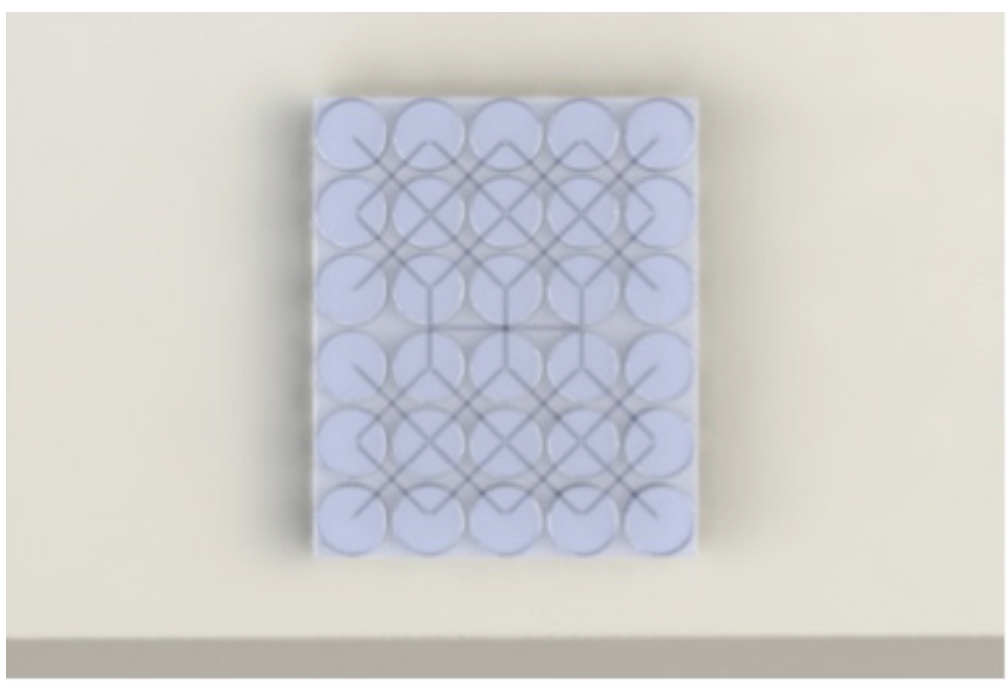

Figura 6. Muestra del sistema de cilindros vacíos que permiten la entrada y salida de aire por medio de los ductos. Fuente: Elaboración propia

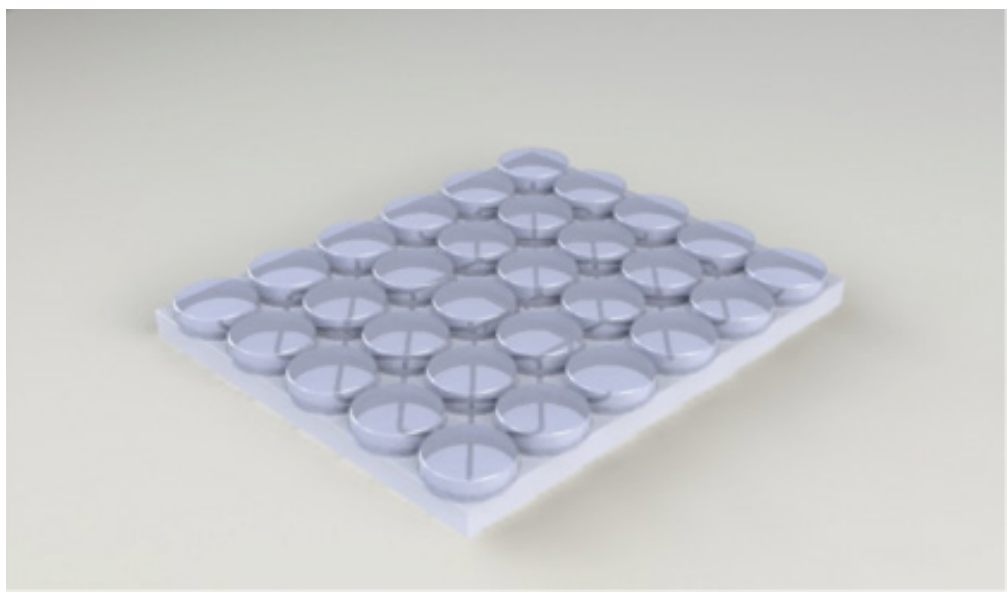


Tercero, el sistema bombeo de aire se alimenta por medio de dos bombas independientes que manejan el flujo de entrada y salida, intercalando la función y generando movimiento. Cada bomba emplea una válvula de escape que permite inflar la mitad de los cilindros de forma combinada.
Cuarto, la simulación de un cuerpo permite señalar los puntos de riesgo sobre la colchoneta en cada una de las posiciones seleccionadas. Estos puntos son tomados de la bibliografía previamente definidos, según estudios anteriormente realizados.

Figura 7. Muestra de la simulación del cuerpo con el cual se tomaron las referencias de los puntos de riesgo. Fuente: Elaboración propia

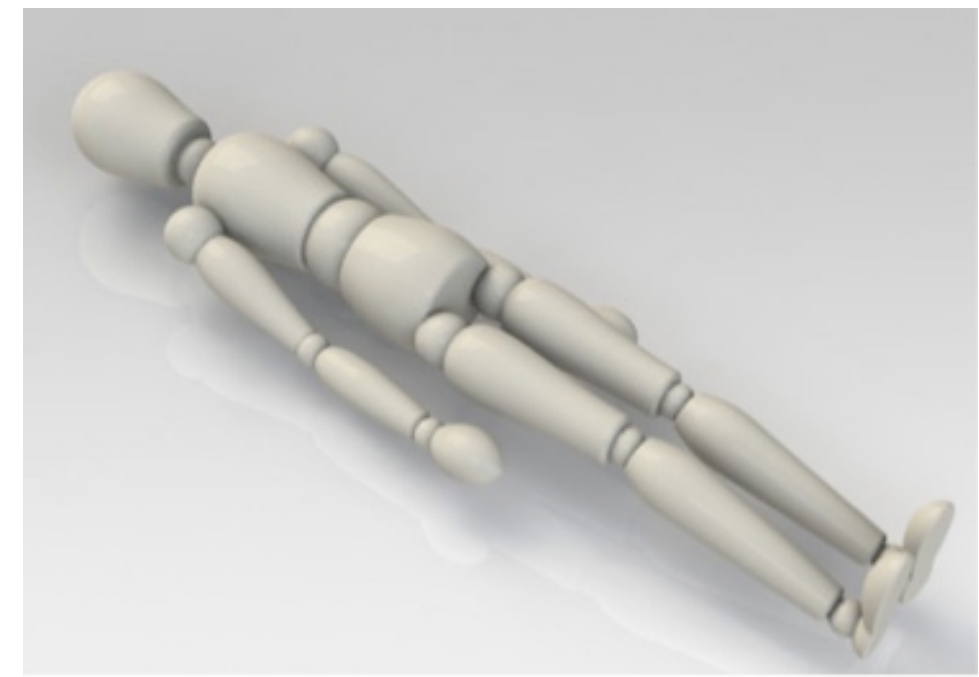

Figura 7. Muestra de la simulación del cuerpo con el cual se tomaron las referencias de los puntos de riesgo. Fuente: Elaboración propia

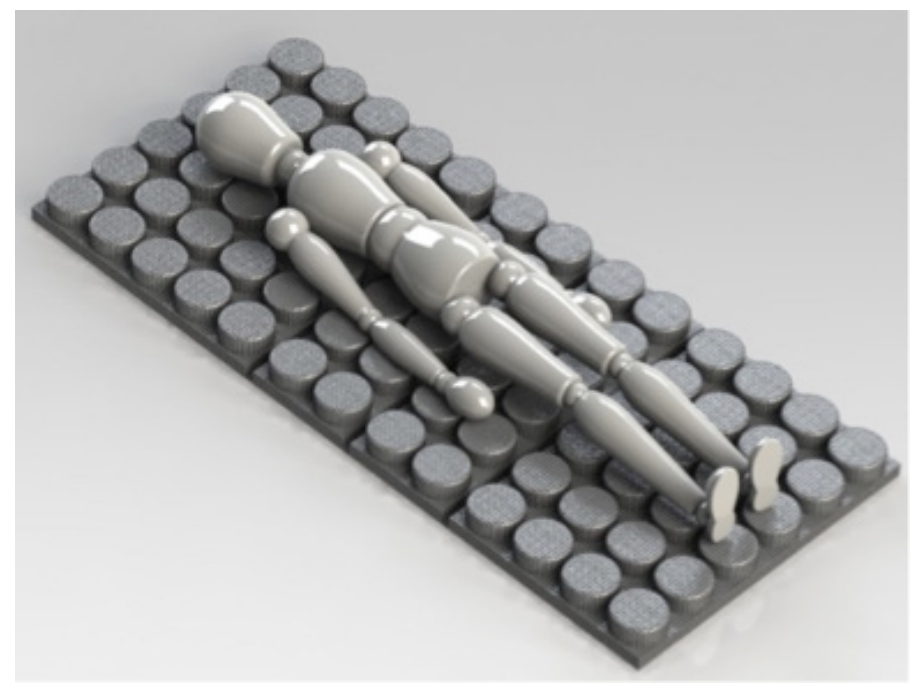


- Selección de variables

Posterior a la ubicación de los puntos de riesgo, se seleccionan las variables que permitirán evaluar el funcionamiento de la colchoneta. Es necesario conocer cómo responde el material frente a la presión ejercida por el peso del cuerpo sobre la superficie en contacto. Asimismo, el comportamiento de la fuerza de reacción producida por la superficie determinará la resistencia de los cilindros.

\section{RESULTADOS}

\section{- Diseño final de la colchoneta}

En la Figura 9 se presenta el diseño final de la colchoneta con la malla generada por el software para la simulación.

Figura 9. Diseño final de la colchoneta. Fuente: Elaboración propia

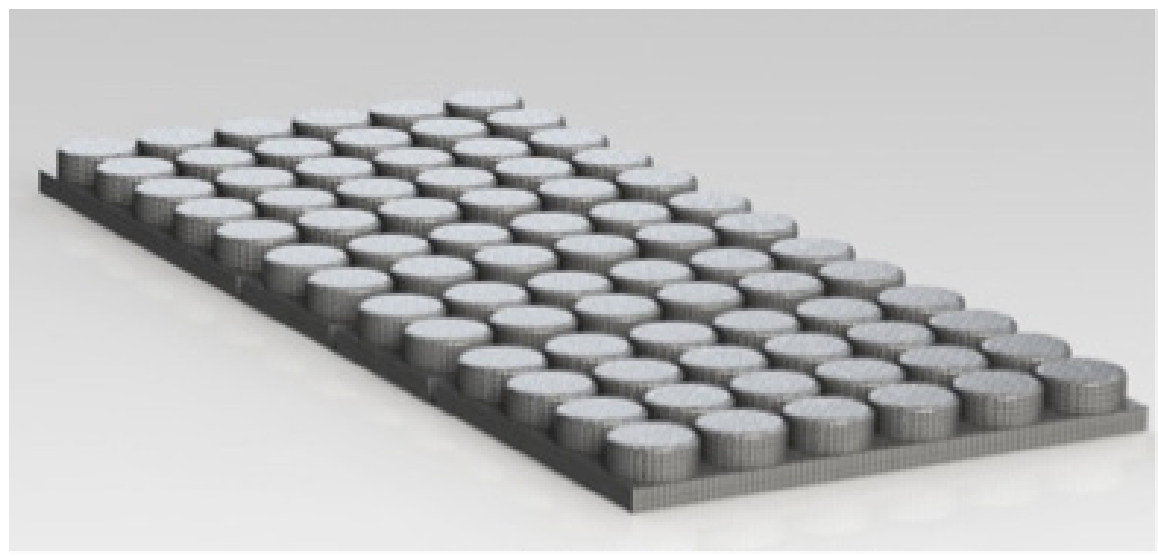

La escala en la que se miden estas variables es Von Mises (Presiones), un criterio de resistencia estática para materiales dúctiles. Este evidencia los resultados en una escala de color que simboliza el máximo, con el color rojo, y el mínimo, con el color azul. De esta manera, en la ejecución de la simulación se evidencia el color rojo cuando el cilindro está expuesto a la máxima presión y disminuye en escala progresiva de color pasando por tonos naranja, amarillo, verde y, por último, azules donde se está expuesto a presiones bajas o nulas. Estas variaciones de color son vistas mientras los cilindros llevan a cabo su proceso de inflado-desinflado.
- Ubicación de los puntos de riesgo en las cuatro posiciones

Las variables medidas por medio de la simulación son fuerza de reacción en los tres componentes, a saber, X (largo de la colchoneta), Y (grosor de la colchoneta) y Z (ancho de la colchoneta); presión mínima y máxima para cada una de las cuatro posiciones: decúbito supino, decúbito lateral, decúbito prono y sentado. La Figura 10 presenta la ubicación de los puntos de riesgo plasmados sobre la colchoneta para las diferentes posiciones mencionadas en anteriores secciones. 
Figura 10. Puntos de riesgo en las 4 posiciones. Fuente: Elaboración propia
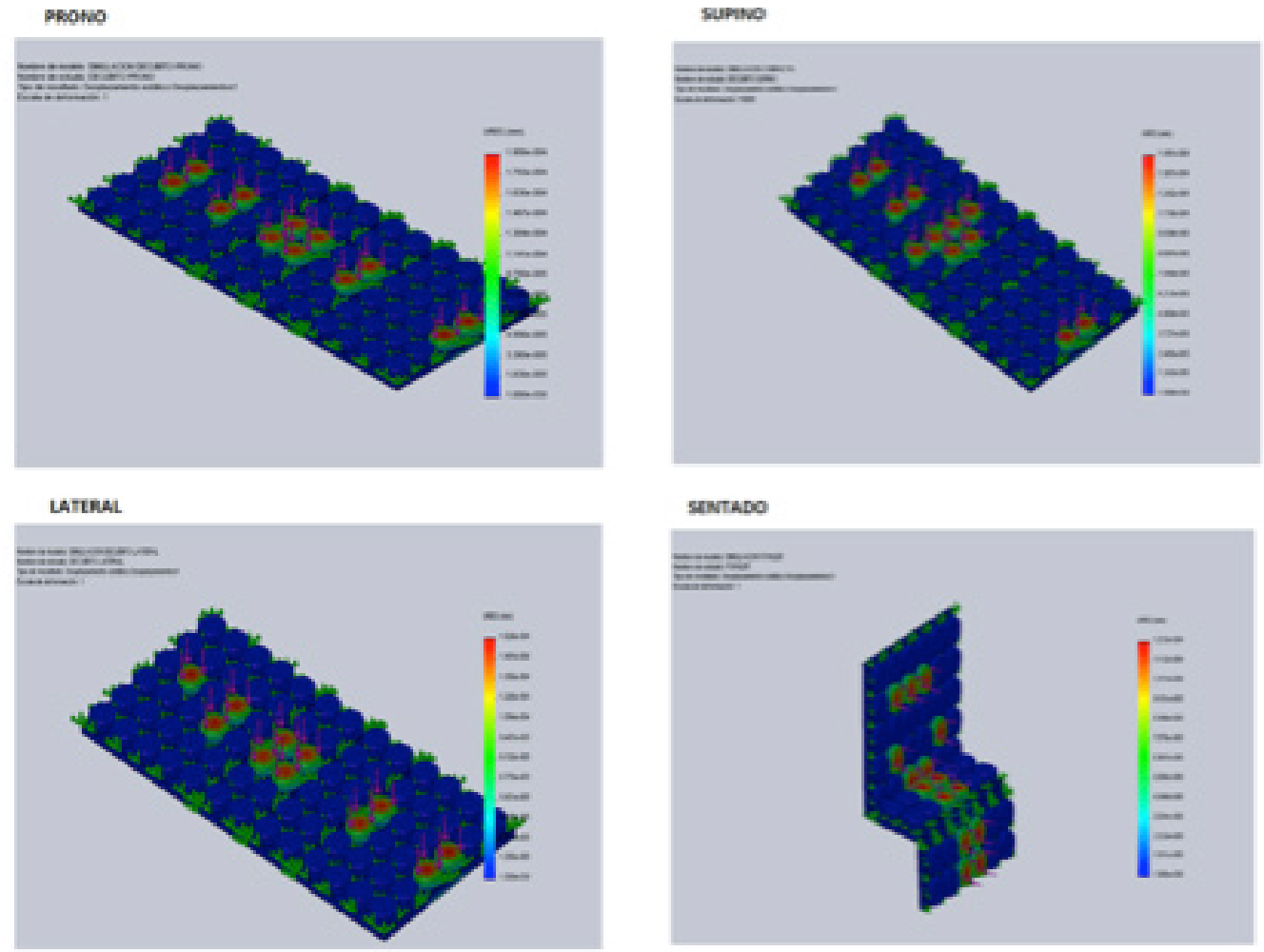

- Medición de variables

\section{Fuerza de reacción}

Cuando el paciente se ubica sobre la colchoneta, el peso corporal realiza una fuerza sobre la superficie en contacto distribuyendo el valor de la magnitud en los tres ejes $X$ (largo de la colchoneta), Y (grosor de la colchoneta) y Z (ancho de la colchoneta) como consecuencia a esta acción se manifiesta

una reacción por parte de la superficie de igual magnitud para cada eje, pero en sentido contrario.

Posteriormente se encontrarán las fuerzas de reacción producidas por la colchoneta a través de la simulación para cada uno de los ejes en las cuatro posiciones seleccionadas, variando el peso corporal.

Tabla 2. Valor del componente X de la fuerza en cada una de las cuatro posiciones variando el peso. Fuente: Elaboración propia

\begin{tabular}{|l|l|l|l|l|}
\hline \multirow{2}{*}{$\begin{array}{l}\text { Peso } \\
\text { Kg }\end{array}$} & \multicolumn{3}{|l|}{ Posición decúbito } & \multirow{2}{*}{ Sentado } \\
\cline { 2 - 5 } & Lateral & Supino & Prono & \\
\hline 50 & $8,97 \mathrm{E}-04$ & $1,06 \mathrm{E}-04$ & $1,82 \mathrm{E}-04$ & $3,12 \mathrm{E}+02$ \\
\hline 70 & $1,24 \mathrm{E}-03$ & $1,45 \mathrm{E}-04$ & $2,46 \mathrm{E}-04$ & $4,35 \mathrm{E}+02$ \\
\hline 100 & $1,79 \mathrm{E}-03$ & $2,11 \mathrm{E}-04$ & $3,64 \mathrm{E}-04$ & $6,56 \mathrm{E}+02$ \\
\hline 150 & $2,63 \mathrm{E}-03$ & $3,15 \mathrm{E}-04$ & $5,39 \mathrm{E}-04$ & $1,06 \mathrm{E}+03$ \\
\hline
\end{tabular}


El peso está expresado en kilogramos (kg) por el uso habitual que la mayoría de personas maneja. Se entiende que el peso es una fuerza y debería expresarse en newton (N). Por lo anterior, las fuerzas de reacción están expresadas en $\mathrm{N}$.

En la Figura 11 se observa que la posición sentado manifiesta una fuerza de reacción considerablemente mayor, en comparación con las otras posiciones. Esta situación se presenta por tener 11 de los 15 puntos de apoyo con la fuerza dirigida en el eje $\mathrm{X}$.

De la misma manera que el eje $X$, el peso está expresado en kg por el uso habitual que la mayoría de personas maneja. También se entiende que el peso es una fuerza y debería expresarse en newton, por lo que las fuerzas de reacción están expresadas en $\mathrm{N}$.

Figura 11. Comportamiento del componente $X$ de la fuerza de reacción para las cuatro (4) posiciones variando el peso. Fuente: Elaboración propia.

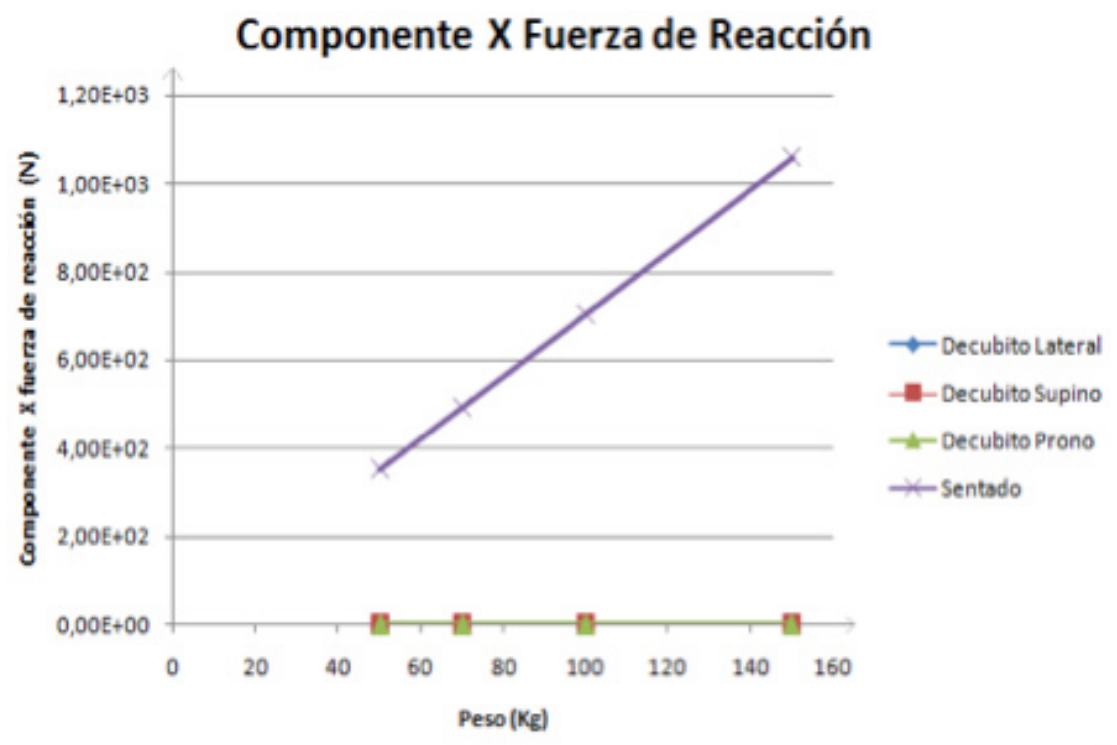

Tabla 3. Valor del componente Y, de la fuerza de reacción, en las cuatro posiciones variando el peso. Fuente: Elaboración propia.

\begin{tabular}{|c|c|c|c|c|}
\hline \multirow{2}{*}{$\begin{array}{l}\text { Peso } \\
\text { kg }\end{array}$} & \multicolumn{3}{|c|}{$\begin{array}{l}\text { POSICIÓN DECUBITO } \\
\text { Posición decúbito }\end{array}$} & \multirow[t]{2}{*}{ Sentado } \\
\hline & Lateral & Supino & Prono & \\
\hline 50 & 486,167 & 486,168 & 486,17 & 177,8 \\
\hline 70 & 680,634 & 680,635 & 680,63 & 250,32 \\
\hline 100 & 972,334 & 972,336 & 972,33 & 323,6 \\
\hline 150 & 1458,5 & $1.458,50$ & $1.458,50$ & 409,9 \\
\hline
\end{tabular}


En la Figura 12 se observa el comportamiento de la fuerza de reacción en el eje Y. Esta corresponde, aproximadamente, a un 99\% de la fuerza ejercida (peso corporal) en las posiciones decúbito. La posición sentado presenta magnitudes menores por tener cuatro de los 15 puntos de apoyo con la fuerza dirigida en el eje $Y$.
Como en el componente $X$ y $Y$, el peso está expresado en $\mathrm{kg}$ dado el uso habitual que la mayoría de personas maneja, se entiende que el peso es una fuerza y debería expresarse en newton. Asimismo, las fuerzas de reacción están expresadas en $\mathrm{N}$.

Figura 12. Comportamiento del componente Y de la fuerza de reacción en las cuatro posiciones variando el peso. Fuente: Elaboración propia.

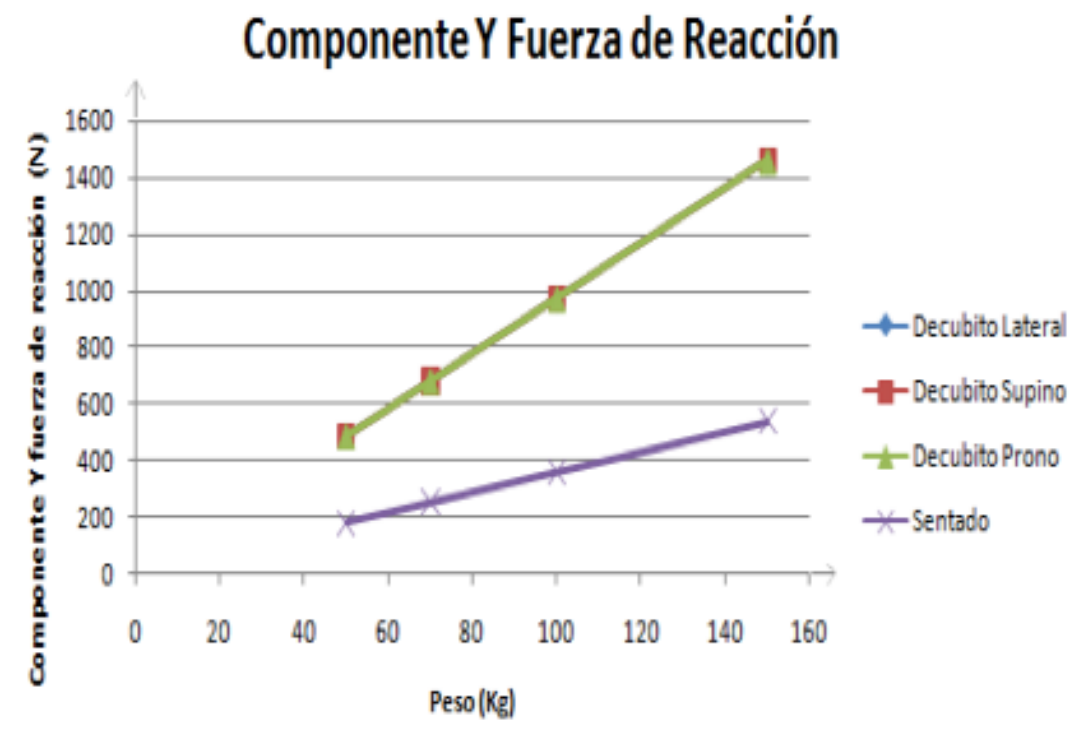

Tabla 4. Valor del componente Z de la fuerza de reacción en las cuatro posiciones variando el peso. Fuente: Elaboración propia.

\begin{tabular}{|c|c|c|c|c|}
\hline \multirow[t]{2}{*}{$\begin{array}{l}\text { Peso } \\
(\mathrm{Kg})\end{array}$} & \multicolumn{3}{|c|}{$\begin{array}{l}\text { POSICIÓN } \\
\text { DECUBITOPosición } \\
\text { decúbito }\end{array}$} & \multirow[b]{2}{*}{ Sentado } \\
\hline & Lateral & \begin{tabular}{|l} 
Supino \\
\end{tabular} & \begin{tabular}{|l|} 
Prono \\
\end{tabular} & \\
\hline 50 & $\begin{array}{l}3,99 \mathrm{E}- \\
05\end{array}$ & $\begin{array}{l}1,73 \mathrm{E}- \\
03\end{array}$ & $\begin{array}{l}2,43 \mathrm{E}- \\
04\end{array}$ & $\begin{array}{l}7,91 \mathrm{E}- \\
04\end{array}$ \\
\hline 70 & $\begin{array}{l}5,52 \mathrm{E}- \\
05\end{array}$ & $\begin{array}{l}2,41 \mathrm{E}- \\
03\end{array}$ & $\begin{array}{l}3,36 \mathrm{E}- \\
04\end{array}$ & $\begin{array}{l}1,10 \mathrm{E}- \\
03\end{array}$ \\
\hline 100 & $\begin{array}{l}\text { 7,99E- } \\
05\end{array}$ & $\begin{array}{l}3,45 \mathrm{E}- \\
03\end{array}$ & $\begin{array}{l}4,86 \mathrm{E}- \\
04\end{array}$ & $\begin{array}{l}1,58 \mathrm{E}- \\
03\end{array}$ \\
\hline 150 & $\begin{array}{l}1,18 \mathrm{E}- \\
04\end{array}$ & $\begin{array}{l}5,17 \mathrm{E}- \\
03\end{array}$ & $\begin{array}{l}7,23 \mathrm{E}- \\
04\end{array}$ & $\begin{array}{l}2,36 \mathrm{E}- \\
03\end{array}$ \\
\hline
\end{tabular}


En la Figura 13 la fuerza de reacción en el eje Z presenta valores mínimos debido a que no tiene puntos de apoyo directos. Por lo tanto, no son significativos para nuestro estudio.

\section{- Presión}

Cuando el cuerpo se ubica sobre una colchoneta su peso ejerce una fuerza perpendicular al área, la magnitud aumenta o disminuye dependiendo de la posición y las protuberancias óseas apoyadas sobre la colchoneta.

El peso está expresado en kg por el uso habitual que la mayoría de personas maneja. Se entiende que el peso es una fuerza y debería expresarse en newton (N). Por lo anterior, las presiones están expresadas en $\mathrm{N} / \mathrm{m}^{\wedge} 2$ (Pascal).

Figura 13. Comportamiento del componente Z de la fuerza de reacción de las cuatro posiciones variando el peso. Fuente: Elaboración propia.

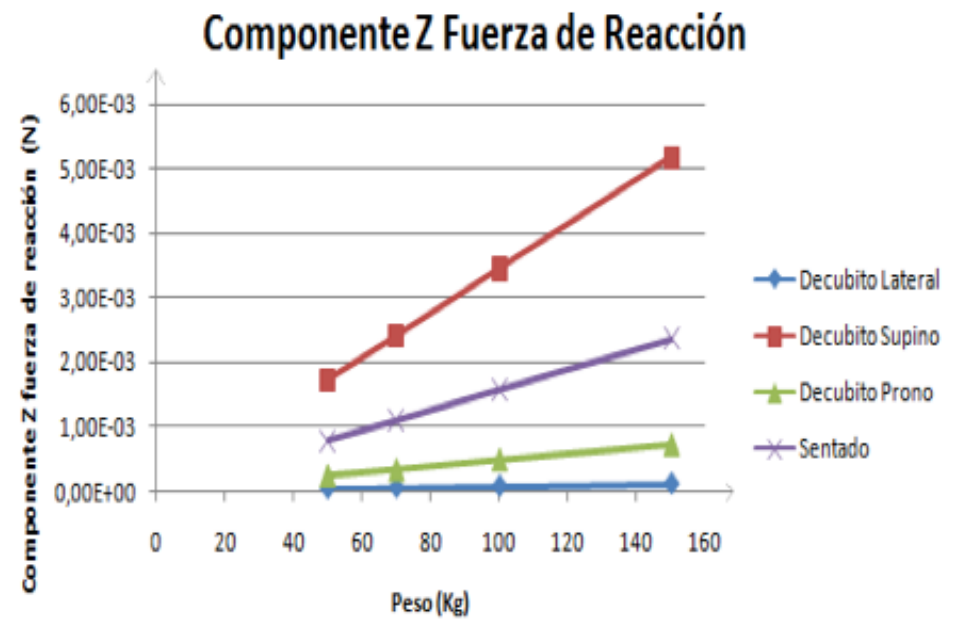

Tabla 5. Valor de la presión mínima en las cuatro posiciones variando el peso. Fuente: Elaboración propia.

\begin{tabular}{|c|c|c|c|c|}
\hline \multirow{2}{*}{$\begin{array}{l}\text { Peso } \\
(\mathrm{Kg})\end{array}$} & \multicolumn{3}{|c|}{$\begin{array}{l}\text { POSICION } \\
\text { DECUBITOPosición } \\
\text { decúbito }\end{array}$} & \multirow[b]{2}{*}{ Sentado } \\
\hline & Lateral & Supino & Prono & \\
\hline 50 & $\begin{array}{l}2,33 \mathrm{E}- \\
04\end{array}$ & $\begin{array}{l}7,75 \mathrm{E}- \\
05\end{array}$ & $\begin{array}{l}6,78 \mathrm{E}- \\
04\end{array}$ & 0 \\
\hline 70 & $\begin{array}{l}3,26 \mathrm{E}- \\
04\end{array}$ & $\begin{array}{l}1,09 \mathrm{E}- \\
04\end{array}$ & $\begin{array}{l}9,50 \mathrm{E}- \\
04\end{array}$ & 0 \\
\hline 100 & $\begin{array}{l}4,66 \mathrm{E}- \\
04\end{array}$ & $\begin{array}{l}1,55 \mathrm{E}- \\
04\end{array}$ & $\begin{array}{l}1,36 \mathrm{E}- \\
03\end{array}$ & 0 \\
\hline 150 & $\begin{array}{l}7,00 \mathrm{E}- \\
04\end{array}$ & $\begin{array}{l}2,33 \mathrm{E}- \\
04\end{array}$ & $\begin{array}{l}2,03 \mathrm{E}- \\
03\end{array}$ & 0 \\
\hline
\end{tabular}


En la Figura 14 se observan las presiones mínimas en cada una de las posiciones. Para la posición decubito lateral, el punto de menor presión son las orejas. Para la posición decubito supino los codos, decubito prono y los pies. Finalmente, en la posición sentado, el punto menor de presión son los gemelos.
En esta ultima posición se presenta presión cero al no existir un contacto con la superficie.

De nuevo, el peso está expresado en kg. Además, se entiende que el peso es una fuerza y se debería expresar en newton $(\mathrm{N})$ por lo que las presiones están expresadas en $\mathrm{N} / \mathrm{m}^{\wedge} 2$ (Pascal).

Figura 14. Comportamiento de la presión mínima en las cuatro posiciones variando el peso. Fuente: Elaboración propia.

\section{PRESION MINIMA}

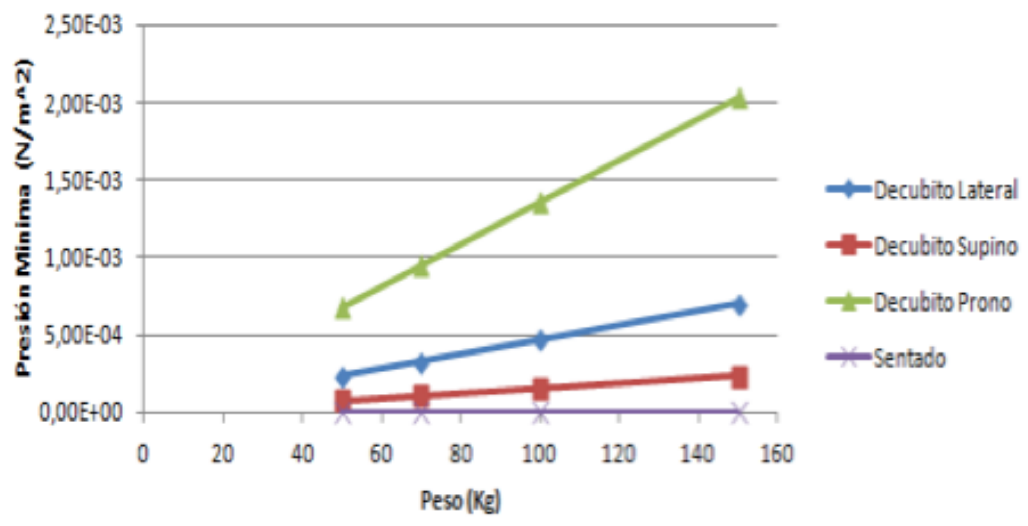

Tabla 6. Valor de la presión máxima en cada una de las cuatro posiciones variando el peso. Fuente: Elaboración propia.

\begin{tabular}{|c|c|c|c|c|}
\hline \multirow{2}{*}{$\begin{array}{l}\text { PESO } \\
(\mathrm{Kg})\end{array}$} & \multicolumn{3}{|c|}{ POSICION DECUBITO } & \multirow[b]{2}{*}{ SENTADO } \\
\hline & LATERAL & SUPINO & PRONO & \\
\hline 50 & 4662,89 & 4274,16 & 4449,3 & 6296,18 \\
\hline 70 & 6528,05 & 5983,82 & 6229,03 & 8814,66 \\
\hline 100 & 9325,78 & 8548,32 & 8898,61 & 12592,4 \\
\hline 150 & 13988,7 & 12822,5 & 13347,9 & 18888,6 \\
\hline
\end{tabular}


En la Figura 15 se observan las presiones máximas en cada una de las posiciones. Para la posición decúbito lateral, el punto de mayor presión son las caderas y acromion; en el decubito supino el sacro; en el decúbito prono las costillas, o mamas en las mujeres, y rodillas. Por último, en la posición sentado el punto de mayor presión son el coxis y los subgluteos, presentando la presión máxima en esta posición.
SolidWorks 2010, con altos niveles de fidelidad, cumpliendo con las expectativas y arrojando datos de alta calidad.

La simulación permite ahorrar tiempo y costos en el diseño. También reduce el número de prototipos de prueba generados antes de iniciar con la producción y ofrece la posibilidad de mejorar la calidad del diseño presentado para así obtener resultados de alta confiabilidad.

Figura 15. Comportamiento de la presión máxima en las cuatro posiciones y masas. Fuente: Elaboración propia.

\section{PRESION MAXIMA}

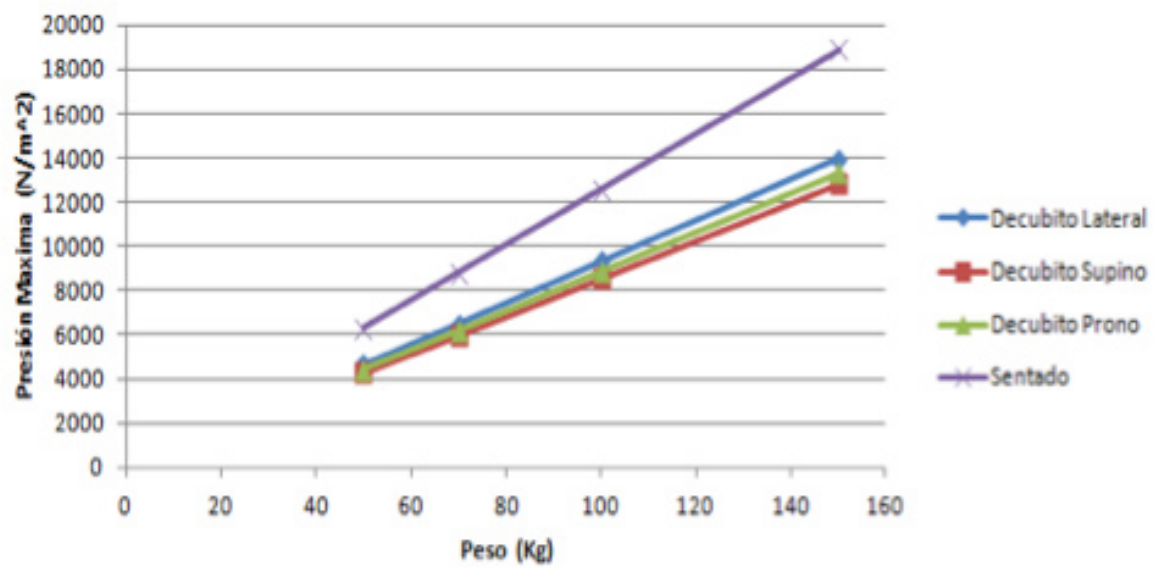

\section{CONCLUSIONES}

Varios mercados en el mundo están trabajando en pro de la calidad de vida de las personas con movilidad reducida. Lo novedoso del presente trabajo es la integración de algunas técnicas descritas. Estas permitieron simular una colchoneta que varía los puntos de apoyo a través de ductos que intercalan la entrada y la salida de aire, dividiéndose en cuatro partes, por medio de cremalleras para mayor versatilidad en su uso.

El desarrollo de la tecnología permite utilizar aplicaciones de la computación como herramienta de trabajo que genera servicios en el área de la salud. Por lo anterior, se representa simultáneamente un sistema colchoneta-paciente por medio de una simulación. Así, se adapta, a la colchoneta, la variación de parámetros tales como material de recubrimiento, pesos corporales, posición del paciente, posición de la colchoneta, áreas de apoyo y flujo de aire. Además, se recrea el comportamiento real que tendría el prototipo por medio del software
La simulación permite tener en cuenta diferentes factores para el diseño, ajustándolos a la normativa como lo es el material de recubrimiento (forro) y dimensiones de la colchoneta. Por otro lado, según lo indica los parámetros de evaluación estándar para la tela utilizada en el forro de la colchoneta, se encontró una variable de interés resistencia mínima en tensión [9].

El comportamiento del material de recubrimiento seleccionado para la simulación es satisfactorio. Las presiones ejercidas no superan el límite de tracción, garantizando un ciclo de vida útil óptimo. Además, el sistema de ductos que alterna los puntos de apoyo con la superficie se adapta en la parte superior de la base de espuma y permite la entrada y salida de aire. También se observa que los ductos de $4 \mathrm{~cm}$ de diámetro permiten el llenado de forma eficiente simulando una bomba de aire de 150 Psi. Por último, en la simulación no se tiene en cuenta el material de estos ductos. Esto dificulta analizar si soportan la presión que circula a través de ellos, también impide tener en cuenta la masa neta de la colchoneta para su difícil o fácil transporte. 


\section{AGRADECIMIENTOS}

Este trabajo fue realizado gracias a la colaboración y apoyo incondicional del Dr. Raúl Narváez Sánchez, Profesor Titular de la Especialización en Bioingeniería de la Universidad de la Distrital Francisco José de Caldas, quien sugirió la idea de la colchoneta y asesoró el desarrollo de la presente investigación aportando conocimientos adquiridos dentro de su labor médica para la elaboración de la simulación.

\section{LISTA DE REFERENCIAS}

[1] Organización Mundial de la Salud, "Discapacidades", 2011. [En línea]. Disponible en: de http:// www.who.int/topics/disabilities/es/

[2] "Identificación de las personas con discapacidad en los territorios desde el rediseño del registro", DANE, 2008. [En línea]. Disponible en: https://www.dane.gov.co/files/investigaciones/ discapacidad/identificacion\%20en\%20los\%20 territorios.pdf

[3] C. Canet, et al., Manual de prevención y tratamiento de las úlceras por presión. España: Hospital Universitario Marqués de Valdecilla, 2003.

[4] I. Bautista y L. Bocanegra, "Prevalencia y factores asociados de las úlceras por presión en pacientes hospitalizados en los servicios de medicina interna, neurología y cuidados intensivos de una institución prestadora de salud de III nivel de la ciudad de Bogotá en el año 2009", trabajo de grado (pregrado), Pontificia Universidad Javeriana, 2009 [En línea]. Disponible en: https://repository.javeriana.edu.co/handle/10554/9848

[5] "Informe de gestión 2002-2010", Min. Protección Social, Bogotá, Colombia, 2010 [En línea]. Disponible en: https://www.minsalud.gov.co/ Ministerio/Documents/Informe\%20Rendici\%C3\%B3n\%20de\%20Cuentas\%202002-2010.pdf

[6] Nimbus professional (2010). Arjohuntleigh, Gentice Group. Recuperado de http://www. arjohuntleigh. com/es/ProductDetails.asp?PageNumber=2502\&ProductCategory_Id=63\&Product_ld $=501$

[7] M, Arcos, et al., "Protocolo de actuación en úlceras por presión", Cirugía General y del aparato digestivo, 2007, pp. 17-18.

[8] Departamento de producción, "Sistema interno de colchones Spring", Colchones Spring, Colombia, 2011.

[9] "Introducción a las aplicaciones de análisis de tensión con SolidWorks simulation, guía del estudiante", Dassault Systemes SolidWorks Corporation, Massachusetts, EEUU 2010 [En línea]. Disponible en: https://docplayer.es/829396-Introduccion-a-las-aplicaciones-de-analisis-de-tension-con-solidworks-simulation-guia-del-estudiante.html

[10] Proyecto de norma técnica colombiana NTC 2094, Icontec (2009) [En línea]. Disponible en: http://es.scribd.com/doc/50119697/NTC2094 